\title{
Las dimensiones de la calidad del servicio en el proceso de distribución y comercialización de energía eléctrica
}

Manuel Enrique Dávila

\section{Bustamante}

Universidad Tecnológica Comisión Federal de Electricidad manuel.davila@cfe.gob.mx

\section{José Ángel Coronado}

\section{Quintana}

Universidad de Sonora coronado@pitic.uson.mx

\section{Bayardo Manuel}

Cerecer Castro

Universidad Tecnológica Comisión Federal de Electricidad bayardo.cerecer@cfe.gob.mx

\section{Resumen}

En este artículo se revisa las dimensiones de la calidad del servicio percibida por los clientes del sistema eléctrico para una investigación a través de un modelo, integrando las teorías de SERVQUAL, calidad de salida y servicio en campo. Así, se aplicó un análisis factorial para evaluar la composición de las dimensiones y el método de ecuaciones estructurales para validar el modelo teórico del caso. La muestra es de 3803 usuarios de los estados de Sonora y Sinaloa de la República Mexicana, la cual se operó por medio de una encuesta telefónica. Los resultados en el análisis factorial mostraron que la composición de las dimensiones de la calidad del servicio funcional o de proceso no coincide con la composición y dimensiones del modelo SERVQUAL; asimismo, el constructo de la calidad funcional en base es diferente al constructo de la calidad funcional de campo y, por último, el análisis de ecuaciones estructurales confirma que el constructo de la calidad de salida no es una dimensión diferente separada de la calidad funcional, por lo que ambas miden la calidad del servicio.

Palabras clave: calidad del servicio, SERVQUAL, calidad funcional, calidad de salida, servicio en campo, encuesta telefónica. 


\title{
Dimensions of service quality in the distribution and commercialization pro- cess of electric energy
}

\begin{abstract}
This article reviews the dimensions of service quality perceived by customers of the electric system for research through a model, which integrates the theories of SERVQUAL, outcome quality and field service. Factor analysis to evaluate the composition of the dimensions and a structural equation method to validate the theoretical model of the case were applied. The sample is of 3,803 users via a telephone survey operated in the states of Sonora and Sinaloa in the Mexican Republic. The factor analysis results show that the composition of the dimensions of functional service quality or process does not match the composition and dimensions of the SERVQUAL model. Likewise, the construct of the functional quality in base is different from the construct of the functional quality in field and, finally, the structural equation analysis confirms that the construct of outcome quality is not a separate dimension from the functional quality, therefore, both measure service quality.
\end{abstract}

Keywords: Service quality, SERVQUAL, functional quality, outcome quality, field service, telephone survey.

\section{Introducción}

Es importante notar que la distinción clave entre un servicio y un producto es el paradigma de que el cliente deriva el valor del servicio a través de un elemento intangible (Lovelock, 2001). En la industria eléctrica, la percepción del servicio involucra la atención de fallas y el mantenimiento preventivo fuera de la percepción del cliente, así como aspectos de incertidumbre, producto de los fenómenos atmosféricos y accidentes provocados por terceras personas. Esto produce mayor complejidad en la medición de la percepción del servicio de energía eléctrica.

En cuanto a la conceptualización y medición de la calidad del servicio, Carman (1990) declara que es un concepto complicado de abordar debido a la característica de intangibilidad que compone al servicio, los problemas en la recepción, la producción en tiempo real y la diferenciación entre la calidad humanística y mecanicista. Tras años de investigación, continúan los debates sobre la conceptualización y medición de la percepción de la calidad del servicio en la literatura (Brady y Cronin, 2001). Esta controversia dificulta la determinación de cómo definir y operacionalizar la calidad del servicio en estudios empíricos. 
La Comisión Federal de Electricidad (CFE) es una empresa paraestatal regida por las estrategias del Gobierno Federal, el cual fomenta la mejora de la calidad de los productos y servicios del sector público, que tienen un alto impacto en la ciudadanía (Modelo intragob, 2005); esto ha promovido que las instituciones de gobierno se vean más como prestadoras de servicio. No obstante, Disend (1991) argumenta que existen razones naturales por las que se da un servicio pobre en el sector público; entre ellas podemos señalar la no existencia de competencia ni motivación por la utilidad financiera, bajo incentivo para tomar riesgos en pro del éxito, tamaño excesivo, burocracia y salarios menores que los del sector privado.

El estudio se ubica en los estados de Sonora y Sinaloa, donde la medición de la calidad del servicio y la satisfacción del cliente es importante porque la sensibilidad de la población a sus servicios es alta debido a los pagos por consumo de energía por temperaturas extremas.

\section{Objetivo}

Este trabajo tiene por objetivo confirmar las dimensiones de la calidad del servicio percibida en el contexto de distribución y comercialización de energía eléctrica a través de la encuesta de satisfacción del cliente Enfoke 2004; para ello se establecieron las siguientes hipótesis de trabajo:

- H1: La composición de las dimensiones de la calidad funcional o de proceso de servicio no coincide con la composición y dimensiones del modelo SERVQUAL.

- H2: La calidad funcional en campo es un factor separado de la calidad funcional en base.

- H3: El constructo de la calidad de salida es una dimensión diferente separada de la calidad funcional.

\section{Supuestos y limitaciones}

Como es común en la literatura de la calidad del servicio, este estudio asume que el término de calidad técnica y calidad de salida son uno. También, dentro de las limitaciones encontradas en esta investigación, hay que considerar que el instrumento de medición de la paraestatal cuenta con sólo seis preguntas, pues se realizó de forma telefónica. 


\section{Desarrollo conceptual}

\section{Calidad del servicio}

Se denomina calidad del servicio a la dirección y grado de discrepancia entre la percepción del cliente y sus expectativas, en términos de las dimensiones de la calidad del servicio que pueden afectar el comportamiento futuro de los consumidores (Parasuraman et al., 1985). Asimismo, se caracteriza por la comparación de la excelencia o superioridad del servicio de los proveedores a juicio del cliente (Zeithaml, 1987); subsecuentemente, Parasuraman et al. (1985 y 1988) argumentan que dicha calidad está en función de la diferencia entre expectativa y desempeño a lo largo de un sistema establecido de dimensiones de calidad.

Según Johnston (1995) es necesario identificar las determinantes o dimensiones para ser capaz de especificar, medir, controlar y mejorar la calidad de servicio percibida por el cliente; sin embargo, el objetivo principal es manipular a ésta; esto es esencial para identificar aspectos que puedan influir potencialmente el juicio general del cliente sobre el servicio. Gronroos (1993) postula que la calidad de servicio percibida por el cliente tiene dos dimensiones: una técnica o dimensión de salida y una relacionada a procesos.

\section{Calidad funcional}

El nivel de calidad percibida de servicio no está determinado solamente por el nivel de desempeño de salida de calidad. Parasuraman et al. (1988) identifican cinco dimensiones que evalúan la calidad en el desarrollo del servicio: a) tangibles, entendida como las facilidades físicas, equipamiento y apariencia del personal; b) confiabilidad, que es la habilidad de desempeñar el servicio prometido exactamente y con formalidad; c) sensibilidad, la cual definen como la buena voluntad para ayudar a los clientes y proveer un servicio oportuno; d) aseguramiento, que comprende el conocimiento y cortesía de los empleados y sus habilidades para inspirar confianza; e) empatía, que abarca la atención y cuidado individualizado que la compañía provee a sus clientes.

En relación con la dimensión de confiabilidad, Boulding et al. (1993) argumentan que mientras la calidad de servicio es multidimensional, la confiabilidad es la dimensión clave en la determinación general de la calidad percibida de servicio. En su modelo dinámico de calidad de servicio, ellos encuentran a la confiabilidad 
como la principal manejadora de la percepción general de la calidad de servicio de los clientes. Esto parece ser verdad en otros estudios, donde también las diferentes dimensiones pueden surgir y suplantar una o más de las cinco originales, o colapsar el número general de dimensiones en un número menor. Parasuraman et al. (1994) mejoran el SERVQUAL y reducen su estructura de cinco a tres factores, permaneciendo intacto el de confiabilidad.

A la comprensión de la dimensión de sensibilidad, Parasuraman et al. (1988) la identifican como la buena voluntad o preparación de los empleados para proveer el servicio, incluyendo la puntualidad en la ejecución, como por ejemplo atender a los clientes rápidamente o responder oportunamente a sus solicitudes o reclamos. La sensibilidad del servicio en campo - es decir, en las instalaciones del cliente o fuera de las instalaciones del proveedor del servicio, como es el caso de estudioes más que un aspecto de puntualidad, sobre todo cuando lo relacionamos con el tiempo de espera entre la puesta de la llamada del cliente y la llegada del ingeniero de servicio a la localidad de dicho cliente (Simmons, 2001). Otra manera de relacionar el aspecto de puntualidad con sensibilidad en el campo técnico de servicio es el tiempo de reparación de la falla, el cual tiene asociado encontrar la causa y el tiempo de espera para hacer las reparaciones del equipo (Kumar y Kumar, 2004).

En este trabajo, la dimensión de aseguramiento (Parasuraman et al., 1988) es básica por las características del servicio, la cual inicialmente fue basada en los atributos de comunicación: credibilidad, seguridad, competencia y cortesía (Parasuraman et al., 1985). Ham et al. (2003) resumen varios componentes asociados con el aseguramiento como la comunicación concerniente a mantener a los clientes informados; la credibilidad que envuelve confianza, creencia, y honestidad; la competencia que significa tener las habilidades requeridas y el conocimiento para desarrollar el servicio; la cortesía que es el respeto y consideración; y la seguridad, que es entendida como estar fuera de peligro o riesgo.

En un análisis factorial, Brady y Cronin (2001) no encuentran el aseguramiento como un factor distinto y por esto no lo incluyen como una dimensión, ni como una modificación de las nueve subdimensiones en su modelo. Estos autores revisaron otros estudios (Babakus y Boller, 1992; Carman, 1990; Dabholkar, 2000; Llosa, 1998; Mels et al., 1997) en los que el aseguramiento fue eliminado debido a un análisis factorial; además, señalan que este concepto depende ampliamente del contexto de la industria en estudio. 
Los investigadores han discutido que la dimensionalidad de la calidad de servicio puede depender sustancialmente de un tipo de servicio (Babakus y Boller, 1992). La investigación de Chowdhary y Prakash (1995) sugiere que un número limitado de factores dominantes puede ser la base alrededor de la cual los clientes evalúan la experiencia del servicio. En el servicio de distribución de energía eléctrica, la importancia de un número limitado de factores puede significar que una dimensión de profesionalismo puede encapsular muchos de los atributos de las dimensiones SERVQUAL, tales como empatía, aseguramiento y tangibles.

\section{Calidad de salida}

La calidad técnica o de salida, según Buttle (1996), se relaciona con el resultado del encuentro del servicio, donde la calidad funcional es considerada como el proceso de entrega del servicio.

De acuerdo con Swartz y Brown (1989), la calidad técnica o de salida se centra en qué servicios son entregados y la evaluación toma lugar después del servicio. La naturaleza de la calidad de salida puede ser diferente de un servicio a otro; por ejemplo, en un ambiente de hospital, la calidad de salida puede referirse a la exactitud del diagnóstico, en conjunto con la entrega del tratamiento apropiado, y en la probabilidad que tiene de alcanzar el resultado deseado (Mangol y Babakus, 1991).

Richards y Allaway (1993) incluyen la dimensión de calidad de salida con las dimensiones de calidad de proceso de SERVQUAL en un estudio de servicio de entrega de pizza. El análisis de regresión fue desarrollado separadamente en un modelo SERVQUAL de 22 elementos, un modelo de calidad de seis elementos y después en un modelo combinado de 28 elementos; sus resultados concluyeron que el modelo combinado - que conjunta procesos y atributos de calidad de salida - es superior a los modelos individuales, pues mostraron que los elementos relacionados solamente al proceso explicaron el $45 \%$ de la variación, mientras que al incluir en el modelo los seis elementos de calidad de salida se obtuvo el $71.5 \%$ de la explicación de la variación.

Powpaka (1996) estudió la calidad de salida en cuatro industrias de servicio como un esfuerzo por determinar y establecer un efecto generalizado. El estudio incluye sólo servicios de tipo base, como bancos, restaurantes, trasporte público y salones de belleza; asimismo, opera la calidad de salida como una dimensión 
de SERVQUAL modificado, además de que no es consistente con la teoría, ya que mide esencialmente la calidad de proceso.

\section{Servicio en campo}

Las investigaciones realizadas sobre la calidad del servicio se han aplicado a los servicios en general y muy poco al servicio en campo (Simmons, 2001). Este tipo de servicio implica que el proveedor realice las actividades de manera remota en instalaciones del cliente o de la misma compañía como los servicios de capacitación o consultoría, o en los que se realiza la instalación, reparación o mantenimiento preventivo de equipos. En este caso se observa este fenómeno en el reparto de recibos, toma de lectura, atención de fallas y mantenimiento de las instalaciones eléctricas.

Es importante considerar que el usuario puede no estar presente durante el servicio y las reparaciones requieran varias horas, limitando el involucramiento del cliente en el curso de la prestación del servicio; esta característica es de gran influencia en el proceso de formación de las expectativas (Zeithaml, Berry y Parasuraman, 1993).

Otra característica importante es la disposición de productos y equipo de la empresa a cargo de los clientes, como medidores e infraestructura en sus terrenos. Según los resultados de Lapierre (1996), basados en un estudio en empresas de telecomunicaciones, los clientes industriales ven a las compañías de servicio de dos formas discretas: como instituciones de producto y como instituciones de servicio, lo que amplía el espectro de evaluación, donde un determinante clave de la calidad percibida es el funcionamiento de un producto en el que se incluye el servicio, que a menudo está más allá del control de la organización (Simmons, 2001).

\section{Modelo de investigación}

De acuerdo con los planteamientos anteriores, se diseñó el modelo particular para la investigación en la figura 1; para ello se consideraron las dimensiones de confiabilidad, sensibilidad y aseguramiento (Parasuraman et al., 1988); la separación entre la calidad de salida y la funcional (Gronroos, 1993); así como la integración del factor del servicio en campo (Simmons, 2001). 
Figura 1

Modelo de investigación

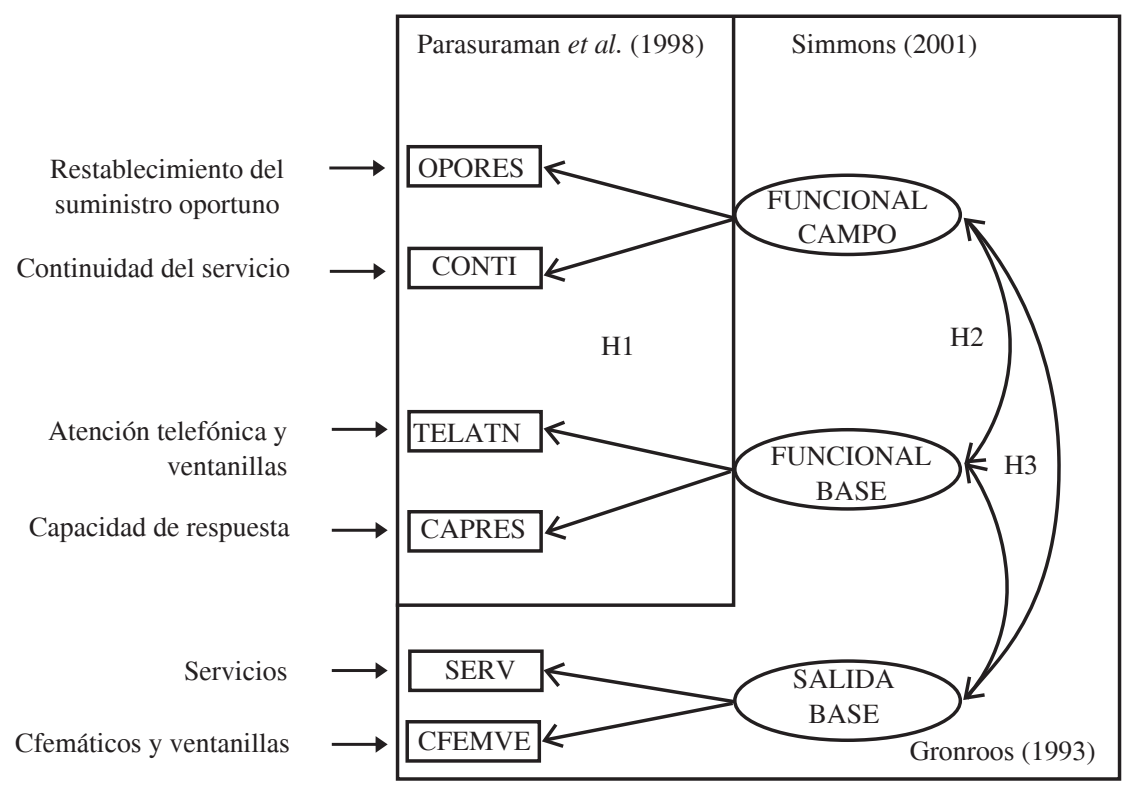

\section{Abordaje metodológico}

\section{Operacionalización de las variables}

Calidad funcional. Una escala modificada de SERVQUAL fue utilizada para operar la calidad funcional. Los elementos se asociaron a las dimensiones funcionales de la confiabilidad, de la sensibilidad y del aseguramiento, centrado en la percepción de los clientes sobre el desempeño de los servicios en campo y base.

La encuesta de satisfacción del cliente del sistema Enfoke (Enfoke Manual, 2004) aplica la escala de Likert de 10 puntos en busca de un acercamiento directo con la desconfirmación, en comparación con el acercamiento computado por la brecha percepción-expectativa de SERVQUAL (Parasuraman et al. 1988), o de la percepción-sola, SERVPERF (Cronin y Taylor, 1992).

Calidad de salida. La calidad de salida está relacionada con el desempeño del resultado final del servicio, ya sea en campo o en base, y se presume una correlación 
con la calidad del servicio percibida. Los elementos asociados con la dimensión técnica o de salida se centran en la percepción de los clientes en el desempeño final del servicio. Sólo se aplicaron a los elementos de servicio de atención en base (centros de atención) dada la naturaleza del tamaño del instrumento, como se observa en el cuadro 1.

\section{Cuadro 1 \\ Instrumento de medición aplicado telefónicamente, relacionado con las variables y dimensiones soportadas teóricamente}

\begin{tabular}{|c|c|c|c|}
\hline \multicolumn{2}{|c|}{ Dimensión } & Variable & Ítem \\
\hline $\begin{array}{l}\overline{\tilde{J}} \\
\stackrel{\overline{0}}{0} \\
\Xi\end{array}$ & Confiabilidad & CONTI & $\begin{array}{l}\text { Después de corregida la falla, ¿su servicio se ha } \\
\text { mantenido estable? } \\
\begin{array}{llllllllll}1 & 2 & 3 & 4 & 5 & 6 & 7 & 8 & 9 & 10 \\
\end{array}\end{array}$ \\
\hline 岂 & Sensibilidad & OPORES & $\begin{array}{l}\text { Cuando se va la luz, ¿se restablece el servicio } \\
\text { oportunamente? Evalúe por favor. } \\
\begin{array}{llllllllll}1 & 2 & 3 & 4 & 5 & 6 & 7 & 8 & 9 & 10\end{array}\end{array}$ \\
\hline 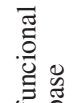 & & TELATN & $\begin{array}{l}\text { ¿Cómo le parece el trato que le damos por teléfono y en } \\
\text { los centros de atención? } \\
\begin{array}{llllllllll}1 & 2 & 3 & 4 & 5 & 6 & 7 & 8 & 9 & 10\end{array}\end{array}$ \\
\hline 节 & Aseguramiento & CAPRES & $\begin{array}{l}\text { ¿Cómo califica la capacidad de respuesta cuando solicita } \\
\text { nuestros servicios? } \\
\begin{array}{llllllllll}1 & 2 & 3 & 4 & 5 & 6 & 7 & 8 & 9 & 10\end{array}\end{array}$ \\
\hline 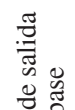 & Resultado del & CFEMVE & $\begin{array}{l}\text { ¿Cómo califica los sistemas de atención que usted utiliza } \\
\text { (cfemáticos, ventanilla electrónica, etc.)? } \\
\begin{array}{ccccccccc}1 & 2 & 3 & 4 & 5 & 6 & 7 & 8 & 9\end{array}\end{array}$ \\
\hline 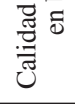 & proceso & SERV & $\begin{array}{l}\text { ¿Cómo califica los servicios que ponemos a su } \\
\text { disposición? } \\
\begin{array}{llllllllll}1 & 2 & 3 & 4 & 5 & 6 & 7 & 8 & 9 & 10\end{array}\end{array}$ \\
\hline
\end{tabular}

\section{Población y muestra}

La población total de la División de Distribución Noroeste de la Comisión Federal de Electricidad (CFE), según el Informe al Consejo de Evaluación de Resultados (2004), es de alrededor de 1420000 clientes; éste es un dato variable en pequeña instancia por las bajas y altas constantes de los usuarios de energía, manteniendo un crecimiento promedio del $4 \%$ desde el 2001.

Según la metodología de Enfoke (2004), el tamaño de la muestra se determinó por medio de la fórmula para la estimación de muestras infinitas en la que no se requiere el tamaño de la población para el cálculo como se muestra en la ecuación 1: 


$$
n=\frac{Z_{\propto / 2}^{2} * P * Q}{e^{2}}
$$

Cada levantamiento se realizó de forma trimestral y en cada ciudad se aplicó una muestra de 120 encuestas, con un nivel de confianza de 90\% y con un error del 7.5\% de los parámetros estimados. Al aplicar el levantamiento del segundo trimestre, el error disminuyó a 5.5\%, de tal forma que para la suma del tercer levantamiento dicho error se estimó en $5 \%$.

En este trabajo se tomaron en cuenta tres trimestres, con excepción del primero que corresponde al periodo de enero a marzo de 2004, porque sólo se aplicaron cinco preguntas. En consecuencia, la muestra estudiada es de 3,803 clientes que corresponden al área geográfica que atiende la División de Distribución Noroeste de la CFE, como se muestra en el cuadro 2.

\section{Cuadro 2}

\section{Muestra por ciudad y entidad federativa}

\begin{tabular}{c|c|c}
\hline Estado & Ciudad & $\begin{array}{c}\text { Muestra } \\
\text { clientes }\end{array}$ \\
\hline Sonora & Nogales & 389 \\
& Caborca & 379 \\
& Hermosillo & 378 \\
& Guaymas & 379 \\
& Cd. Obregón & 378 \\
& Navojoa & 378 \\
\hline Sinaloa & Los Mochis & 383 \\
& Guasave & 378 \\
& Culiacán & 383 \\
& Mazatlán & 378 \\
\hline & Total & 3803 \\
\hline
\end{tabular}

Fiabilidad y validez

Fiabilidad. Para la consistencia interna de la encuesta, se obtuvo el alpha de Cronbach; se considera como valor aceptable un coeficiente igual o superior a 0.70 (Hair et al., 1998).

Validez de contenido. La capacidad del instrumento para recoger el contenido y el alcance del constructo y de la dimensión en esta investigación se apoya en la 
fundamentación teórica y las evidencias empíricas que sostienen la revisión de la literatura realizada.

Validación convergente. Para demostrar que las mediciones están relacionadas se realizó un análisis factorial de acuerdo con Parasuraman et al. (1994) y Hernández et al. (2006), quienes la recomiendan por ser una evaluación más estricta.

Validación discriminante. Para facilitar la interpretación de los constructos se hace referencia a la propiedad de que la medida utilizada no se correlaciona demasiado con las medidas de otros constructos con los que se supone que teóricamente difiere.

\section{Metodología de análisis de datos}

Análisis factorial. Se realizó un análisis factorial a través de SPSS 16 para determinar la estructura de factores del modelo de calidad del servicio en el proceso de distribución y comercialización de energía eléctrica y probar las hipótesis uno y dos. De acuerdo con Felh (2006), el modelo de análisis se realizó en dos etapas: en la etapa uno se estableció la medición del modelo y en la etapa dos se examinaron las hipótesis de las relaciones estructurales entre las variables latentes.

El propósito del análisis factorial fue determinar la estructura de factores y resolver si las preguntas miden las dimensiones de la calidad funcional y calidad de salida como se anticipó en la teoría y de esta forma validar estos constructos; es importante mencionar que para validarlos se previó que miden conceptos diferentes, por lo que se aplicó la rotación oblicua Promax para obtener un patrón claro que proporcione e indique la validación discriminante.

Modelo de ecuación estructural. El modelo de investigación propuesto fue probado utilizando el modelo de ecuaciones estructurales (MEE) en AMOS16, estimando los parámetros y evaluando el ajuste del modelo. Se determinó para cada variable el indicador de la carga de la regresión con su respectivo factor. Los patrones y cargas de la regresión fueron establecidos por la medición del modelo estructural. El modelo de ecuación estructural aplicado contempló la correlación entre los términos de error para algunas de las variables; la correlación y no correlación de los términos de error se ejecutaron basados en el modelo de regresión, aplicando los indicadores de modificación para obtener un modelo efectivo. 


\section{Resultados}

Antes de evaluar el modelo de investigación y las hipótesis individuales, se evaluó la consistencia interna con el alpha de Cronbach de los siete elementos de la encuesta de satisfacción al cliente, que reportó un 0.797 como resultado.

\section{Hipótesis 1}

Se determinó la pertinencia del análisis factorial a través de la evaluación de las correlaciones entre las variables las cuales se encontraron significativas; también se evaluó la prueba anti-imagen observándose que las correlaciones eran menores a la diagonal como se muestra en el cuadro 3. La prueba de esfericidad de Barltlett's fue significativa, pues resultó una chi-cuadrada de 4,891.9 con 15 grados de libertad.

\section{Cuadro 3}

Evaluación de la conveniencia del análisis factorial: correlaciones y correlaciones parciales entre las variables

\begin{tabular}{c|c|c|c|c|c|c|c}
\hline & Variable & TELATN & SERV & CFEMVE & CAPRES & OPORES & CONTI \\
\hline Correlación & TELATN & 1.000 & 0.335 & 0.355 & 0.436 & 0.301 & 0.269 \\
& SERV & & 1.000 & 0.380 & 0.408 & 0.314 & 0.313 \\
& CFEMVE & & & 1.000 & 0.408 & 0.208 & 0.229 \\
& CAPRES & & & & 1.000 & 0.343 & 0.336 \\
& OPORES & & & & & 1.000 & 0.530 \\
& CONTI & & & & & & 1.000 \\
\hline Correlación & TELATN & 0.832 & & & & & \\
Anti-imagen & SERV & -0.111 & 0.842 & & & & \\
& CFEMVE & -0.171 & -0.214 & 0.809 & & & \\
& CAPRES & -0.253 & -0.189 & -0.221 & 0.813 & & \\
& OPORES & -0.110 & -0.106 & 0.015 & -0.115 & 0.735 & \\
& CONTI & -0.042 & -0.105 & -0.042 & -0.110 & -0.437 & 0.739 \\
\hline
\end{tabular}

Se utilizó el método de componentes principales para extraer los factores a través de la prueba de comunalidades mayores a uno, en donde se obtuvo que los seis elementos son mayores a 0.607 . Los factores no rotados resultantes de los seis elementos, incluyendo los dos de la calidad del servicio funcional basada en las dimensiones de SERVQUAL, resultaron de la matriz de componentes constituida por tres factores que explican el $73.24 \%$ de la varianza. El método de rotación aplicado oblicuo (Promax) arrojó una matriz de componentes rotados de tres factores como se muestra en el cuadro 4. 
Los resultados mostraron que los elementos de oportunidad en el restablecimiento (OPORES) y la continuidad (CONTI) componían un solo factor, mezclando las dimensiones de confiabilidad y sensibilidad de SERVQUAL; los elementos de trato en telefónica y centros de atención (TELATNV) y capacidad de respuesta (CAPRES) resultaron integrar un solo factor, mezclando las dimensiones de aseguramiento y sensibilidad. Dados los resultados anteriores y con el objeto de dar respuesta a la hipótesis uno - relativa a conocer si la composición de las dimensiones de la calidad del servicio funcional o de proceso de la encuesta de satisfacción del cliente Enfoke coincidía con la composición del instrumento SERVQUAL — se rechazó la hipótesis nula.

\section{Hipótesis 2}

La hipótesis dos buscó demostrar la separación de la calidad funcional en campo y la calidad funcional en base, para lo cual los resultados del análisis factorial, que se aprecia en el cuadro 4, muestran que los elementos relacionados al campo, como la oportunidad de respuesta (OPORES) y la continuidad (CONTI), constituyen un solo factor, y los elementos que representan la calidad del servicio en los centros de atención personalizada (Calidad del servicio en base) como telefónica y centros de atención (TELATN) y capacidad de respuesta (CAPRES) conformaron otro factor; de esta manera se demostró que la dimensión de la calidad funcional en base es un factor diferente de la calidad funcional en campo.

\section{Cuadro 4 \\ Resultado del análisis factorial}

\begin{tabular}{|c|c|c|c|c|c|c|c|c|c|}
\hline \multirow[b]{3}{*}{ Variable } & \multicolumn{9}{|c|}{ Matriz } \\
\hline & \multicolumn{3}{|c|}{ Componentes } & \multicolumn{3}{|c|}{$\begin{array}{l}\text { Componentes rotados } \\
\text { Método Promax }\end{array}$} & \multicolumn{3}{|c|}{ Estructura } \\
\hline & 1 & 2 & 3 & 1 & 2 & 3 & $\begin{array}{c}\text { Funcional } \\
\text { Campo }\end{array}$ & $\begin{array}{c}\text { Funcional } \\
\text { Base }\end{array}$ & $\begin{array}{l}\text { Salida } \\
\text { Base }\end{array}$ \\
\hline TELATN & 0.666 & -0.257 & -0.597 & 0.006 & -0.094 & 0.971 & 0.313 & 0.384 & 0.927 \\
\hline SERV & 0.682 & -0.176 & 0.484 & 0.150 & 0.865 & -0.182 & 0.422 & 0.834 & 0.295 \\
\hline CFEMVE & 0.629 & -0.477 & 0.241 & -0.191 & 0.794 & 0.165 & 0.176 & 0.800 & 0.486 \\
\hline CAPRES & 0.739 & -0.202 & -0.142 & 0.116 & 0.328 & 0.503 & 0.422 & 0.619 & 0.705 \\
\hline OPORES & 0.664 & 0.560 & -0.044 & 0.862 & -0.068 & 0.088 & 0.866 & 0.311 & 0.359 \\
\hline CONTI & 0.659 & 0.559 & 0.076 & 0.864 & 0.041 & -0.039 & 0.866 & 0.359 & 0.287 \\
\hline
\end{tabular}

Medición de la adecuación de la muestra de Kaiser-Meyer-Olkin (KMO) de : 0.793 


\section{Hipótesis 3}

Para probar la hipótesis tres, se estableció un modelo de competencia aplicando el modelo de ecuaciones estructurales, que se desarrolló con base en los constructos de la calidad funcional de base y campo, y el de calidad de salida, enlazados por flechas de relación.

La evaluación del modelo teórico arrojó los siguientes resultados: el ratio de verosimilitud del estadístico chi-cuadrada tiene un valor de 8.622, con nivel de significancia estadística de 0.071 , por encima del nivel mínimo de 0.05 , pero no por encima de los niveles más conservadores de 0.10 o 0.20 . Este estadístico muestra evidencia de que las matrices efectivas y previstas no son significativas, lo que indica un ajuste aceptable del modelo. El indicador normal de ajuste (NFI) es de 0.998; el coeficiente Tuker-Lewis (TLI), de 0.996; y el índice de comparación de ajuste (CFI), de 0.999. De acuerdo con Hair et al. (1998) los valores del NFI, del TLI y del CFI son aceptables, pues reportan un valor mayor a 0.900. Por lo que respecta a la raíz cuadrada de la media del cuadrado de los residuos (RMSEA) se obtuvo un valor de 0.017 menor a 0.05 considerándose aceptable, según los mismos autores.

Al realizar la comparación del modelo teórico con el modelo unidimensional o de un solo factor, se encontró que los indicadores de ajuste del modelo propuesto (multidimensional) son de mayor aceptación, como se observa en el cuadro de resultados de la figura 2. 
Figura 2

Modelo de análisis factorial confirmatorio de las estimaciones estandarizadas de las correlaciones entre los constructos de la calidad de salida y la calidad funcional del servicio

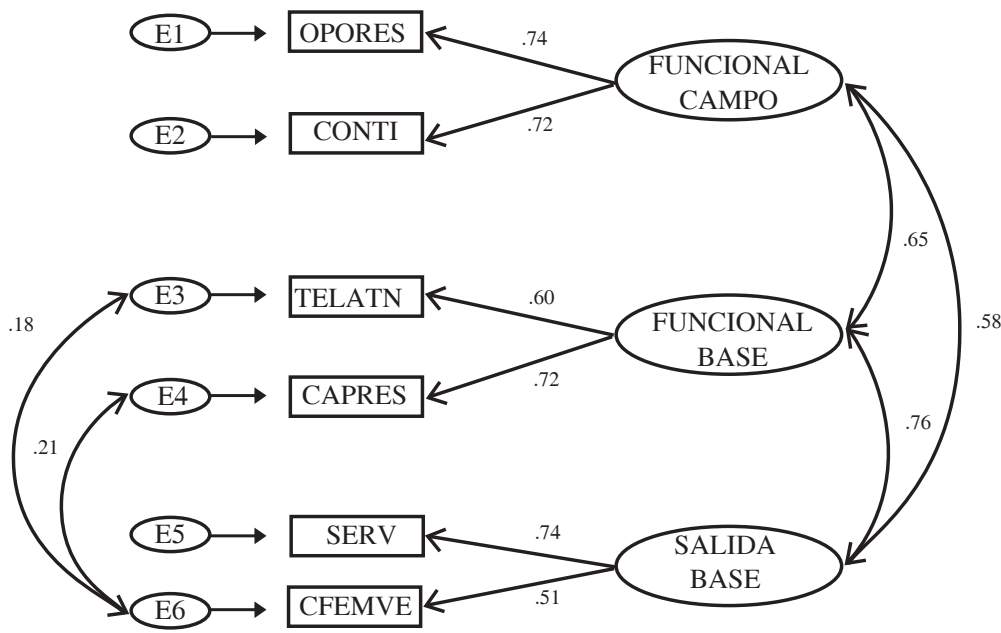

\begin{tabular}{c|c|c|c|c|c|c|c}
\hline Modelo & $\boldsymbol{x}^{2}$ & GL & $\mathbf{P}$ & NFI & TLI & CFI & RMSEA \\
\hline Teórico & 8.622 & 4 & 0.071 & 0.998 & 0.996 & 0.999 & 0.017 \\
Unidimensional & 531.3 & 7 & 0.000 & 0.891 & 0.770 & 0.893 & 0.140 \\
\hline
\end{tabular}

\section{Discusión sobre resultados}

La no confirmación de la composición de las dimensiones de SERVQUAL de Parasuraman (1994) puede estar relacionada a que algunos estudios no pudieron replicar las cinco dimensiones de la calidad encontradas en la investigación original, dando lugar a otras dimensiones (O’Neill y Palmer, 2003). Asimismo, el instrumento aplicado no contempla de forma completa ni las cinco dimensiones ni sus elementos. No obstante, Cronin y Taylor (1992) discuten y expresan la preocupación por la construcción de la escala del modelo SERVQUAL y si los cuestionamientos individuales describen realmente los cinco componentes separados de la calidad del servicio.

La estructura de factores mostró la separación entre campo y base; no obstante que la ubicación de la pregunta sobre capacidad de respuesta puede ser asociada a campo, dicha pregunta tiene una gran trascendencia en la rápida respuesta de las 
llamadas telefónicas (Felh, 2006), como sucede en el centro de atención telefónica al atender una solicitud de servicio o una atención de falla.

En el análisis para probar que la calidad de salida es un constructo diferente separado en la medición de la calidad del servicio percibido, como lo afirman Kang y James (2004), al separar la calidad funcional de la de salida en la evaluación de calidad general percibida de servicio, así como su impacto en la satisfacción del cliente, encontramos que el análisis factorial realizado valida el factor con sus cuestionamientos de cfemáticos y ventanilla, y los servicios de atención.

La determinación de si la calidad de salida es un constructo separado y diferente de la calidad funcional, se operacionalizó a través de un análisis factorial confirmatorio, aplicando el modelo de ecuaciones estructurales validado con los indicadores de ajuste del modelo; así, se encontró que la calidad de salida está correlacionada por encima de 0.50 a los constructos de la calidad funcional. Este resultado está de acuerdo con Richards y Allaway (1993), quienes concluyen que combinando procesos y atributos de calidad de salida en el modelo es superior a los modelos individuales.

Lo anterior coincide con lo señalado por Felh (2006), quien afirma que una alta correlación entre los constructos da pie a un constructo de segundo orden orientando la existencia del constructo de calidad del servicio, así como con lo afirmado por Llosa et al. (1998), quienes aseveran que la calidad de servicio percibida tiene dos grandes facetas: el proceso de servicio y la calidad de salida.

\section{Conclusiones e implicaciones}

Primero, basados en el análisis factorial, la composición de las dimensiones de la calidad del servicio funcional o de proceso no coincide con la composición y dimensiones del modelo SERVQUAL.

Segundo, los resultados del análisis factorial mostraron que el constructo de la calidad funcional base es diferente al constructo de la calidad funcional de campo para este caso de estudio.

Tercero, los resultados del análisis de ecuaciones estructurales, expuesto en la figura 1, confirmaron que el constructo de la calidad de salida no es una dimensión diferente separada de la calidad funcional. 
Una conclusión importante es que la encuesta operada de manera telefónica para un proceso de servicio multidimensional, como el de la CFE, podría no ser demostrativa dado el limitado número de elementos que compone la encuesta.

Este estudio contribuye teórica, metodológica y en resultados generales a la literatura de la calidad del servicio al desarrollar un modelo de calidad del servicio conformado por las dimensiones de calidad funcional, calidad de salida en el ambiente del proceso de distribución de energía eléctrica.

\section{Limitaciones del estudio y recomendaciones}

Esta investigación se enfoca en la evaluación de los servicios desde la perspectiva del instrumento de evaluación Enfoke, dejando fuera otros constructos importantes como valor percibido, lealtad del cliente, intenciones de compra, intenciones de recomendación, imagen percibida, calidad total percibida, etcétera.

Esto permitirá conocer de manera amplia la percepción del cliente y correlacionar los constructos permitiendo a la organización, modificar sus procesos en pro de la mejora del servicio.

Futuras investigaciones deberán buscar contemplar en sus modelos las dimensiones y constructos expuestos en las limitaciones. Asimismo, ampliar la diferenciación entre los constructos del servicio en ambiente de campo y base en función de la calidad del servicio. También buscar la aplicación de encuestas en campo y correo electrónico.

\section{Referencias}

Babakus, E. y G. W. Boller (1992). An empirical assessment of the SERVQUAL scale. Journal of Business Research (24): 253- 268.

Boulding, E., A. Kalra, R. Staelin y V. A. Zeithaml (1993). A dynamic process model of service quality: From expectations to behavioral intentions. Journal of Marketing Research (30): 7-27.

Brady, M. K. y Jr. J. J. Cronin (2001). Some new thoughts on conceptualizing perceived service quality: A hierarchical approach. Journal of Marketing (65): 34-49. 
Buttle, F. (1996). SERVQUAL: Review, critique, research agenda. European Journal of Marketing (30): 8-32.

Carman, J. M. (1990). Consumer perceptions of service quality: An assessment of the SERVQUAL dimensions. Journal of Retailing (66): 33-55.

Chowdhary, C. y M. Prakash (1995). Service quality revisiting the two factor theory. Journal of Services Research (5): 61-75.

Cronin, Jr. J. J. y S. A. Taylor (1992). Measuring service quality: A reexamination and extension. Journal of Marketing (56): 55-68.

Dabholkar, P. A., C. D. Shepherd y D. I. Thorpe (2000). A comprehensive framework for service quality: An investigation of critical conceptual and measurement issues through a longitudinal study. Journal of Retailing (76): 139-173.

Disend, J. E. (1991). How to provide excellent service in any organization. Pensylvania: Chilton Book Company.

Enfoke Manual (2004). Documento institucional. Comisión Federal de Electricidad, Distribución Noroeste.

Fehl, S.(2006). The Relationship of services quality to customer satisfaction: An analysis within industrial business to business technical field service. Universidad de Capella.

Gronroos, C. (1993). Toward a third phase in service quality research: Challenges and future directions. En T. A. Swartz, D. E. Bowen y S. W. Brown (Eds.), Advances in Services Marketing and Management, Greenwich: JAI Press, 2: 49-64.

Hair, J. F. Jr., R. E. Anderson, R. L. Tatham y W. C. Black (1998). Análisis multivariante. 5a. ed., NJ: Prentice Hall.

Ham, C. L. W. Johnson, A. Weinstein, R. Plank y P. Johnson (2003). Gaining competitive advantage: Analyzing the gap between expectations and perceptions of service quality. International Journal of Value - Based Management (16): 197-203. 
Hernandez, R., C. Fernandez y P. Baptista (2006) Metodología de la investigación. 4a. ed., México: McGraw-Hill Interamericana.

Informe al consejo de evaluación de Resultados (2004). Documento institucional. Comisión Federal de Electricidad, Distribución Noroeste.

Johnston, R. (1995). The determinants of service quality: Satisfiers and dissatisfies. International Journal of Service Industry Management (6): 53-71.

Kang, G. y J. James (2004). Service quality dimensions: An examination of Gronroos's service quality model. Managing Service Quality (14): 266277.

Kumar, R., y U. Kumar (2004). A conceptual framework for the development of a service delivery strategy for industrial systems and products. Journal of Business \& Industrial Marketing (19): 310-319.

Lapierre, J. (1996). Service quality: The construct, its dimensionality, and its measurement. En T. A. Swartz, D. E. Bowen y S. W. Brown (Eds.), Advances in Services Marketing and Management, Greenwich: JAI Press, 5: 45-70

Llosa, S., J. Chandon y C. Orsingher (1998). An empirical study of SERVQUAL's dimensionality. Service Industries Journal (18): 16-44.

Lovelock, C. H. (2001). Services marketing: people, technology, strategy. (4a. ed., NJ: Prentice Hall.

Intragob, modelo (2005). Documento institucional. Secretaría de Economía, de Noviembre de 2010 en http://www.spc.gob.mx/materialDeApoyo/redRH/ capacidades/Modelo_Intragob.pdf.

Mangold, G. W. y E. Babakus (1991). Service quality: The front-stage perspective vs the backstage perspective. Journal of Services Marketing (5): 59-79.

Mels, G., C. Boshoff y D. Nel (1997). The dimensions of service quality: The original European perspective revisited. Service Industries Journal (17): 173-189. 
O'Neill, M. y A. Palmer (2003). An exploratory study of the effects of experience on consumer perceptions of the service quality construct. Managing Service Quality (13): 187-196.

Parasuraman, A., V. A., Zeithaml y L. L. Berry (1985). A conceptual model of service quality and its implications for future research. Journal of Marketing (49): 41-50.

(1988). SERVQUAL: A multiple-item scale for measuring consumer perceptions of service quality. Journal of Retailing (64): 12-40.

(1994). Reassessment of expectations comparisons as a comparison standard in measuring service quality: Implications for further research. $J o$ urnal of Marketing (58): 11-124.

Powpaka, S. (1996). The role of outcome quality as a determinant of overall service quality in different categories of services industries: An empirical investigation. Journal of Services Marketing (10): 5-25.

Richard, M. D. y A. W. Allaway (1993). Service quality attributes and choice behavior. Journal of Services Marketing (7): 59-68.

Simmons, D. E. (2001). Field service management: A classification scheme and study of server flexibility, Doctoral dissertation, State University of New York at Binghamton.

Swartz, T. A. y S. W. Brown (1989). Consumer and provider expectations and experiences in evaluating professional service quality. Journal of the Academy of Marketing Science (17): 189-195.

Zeithaml, V. A. (1987). Defining and relating price, perceived quality, and perceived value. Report No. 87-101. Cambridge, MA: Marketing Science Institute.

L. L. Berry y A. Parasuraman (1993). The nature and determinants of customer expectations of service. Journal of the Academy of Marketing Science (21): 1-12. ( 


\section{Anexo}

\section{Estadísticos descriptivos}

\begin{tabular}{l|l|c|c|c}
\hline & \multicolumn{1}{|c|}{ Variable } & N & Media & Desv. típ. \\
\hline Servicio & SERV & 3803 & 1.28 & .764 \\
\hline Cfemativos y ventanilla & CFEMVE & 3803 & 1.39 & .946 \\
\hline Capacidad de respuesta & CAPRES & 3803 & 1.32 & .891 \\
\hline Restablecimiento oportuno & OPORES & 3803 & 1.46 & 1.001 \\
\hline Continuidad del suministro & CONTI & 3803 & 1.27 & .761 \\
\hline
\end{tabular}


